\title{
LA GESTIÓN TRIBUTARIA LOCAL EN ESPAÑA: ALGUNAS PROPUESTAS SOLIDARIAS DEL ESTADO DEL BIENESTAR ANTE LA CRISIS GLOBAL
}

\author{
PUBLIC LOCAL TAX MANAGEMENT IN SPAIN: SOME SOLIDARITY \\ PROPOSALS FOR WELFARE STATE TO THE GLOBAL CRISIS
}

Irene Belmonte Martín

Universidad Miguel Hernández, Elche. España/Spain

irene.belmonte@umh.es

Recibido/Received: 20/12/2012

Aceptado/Accepted: 29/01/2013

\section{RESUMEN}

El gobierno local es un pilar fundamental en el desarrollo del Estado del Bienestar según el principio de máxima cercanía al ciudadano (subsidiariedad). Sin embargo, los municipios se encuentran con múltiples problemas para hacer posible este reto, entre ellos, precisamente su limitada capacidad financiera y el infra municipalismo. Por ello, han tenido que idear nuevas fórmulas administrativas para maximizar la eficacia de su gestión tributaria y recaudación. Estas fórmulas, que incorporan elementos tanto de la Nueva Gestión Pública (NGP) como de la Gobernanza, se han reflejado en distintos modelos que van desde la cooperación inter y supra municipal organizando las competencias desde el ámbito provincial hasta la cesión de gran parte de sus competencias en manos privadas. Con este estudio se pretende explicar las circunstancias de las Haciendas Locales en las que resulta beneficiosa la gestión supramunicipal de los ingresos tributarios para que, en aras de los principios de solidaridad, redistribución y equidad, los municipios puedan maximizar los recursos necesarios para el efectivo desarrollo de sus Políticas Sociales en un Estado del Bienestar que se nos aparece cada vez más incierto.

\section{PALABRAS CLAVE}

Gestión tributaria local, gobernanza, solidaridad, equidad, suficiencia financiera.

\section{SUMARIO}

1. La necesidad de recaudar tributos locales. 2. La gestión de los tributos municipales.3. El panorama de la gestión tributaria local. 4. El horizonte de la gestión tributaria local en España. 5. La modernización de la gestión tributaria local como sustento al Estado del Bienestar. 6. Conclusiones. Bibliografia.

\footnotetext{
ABSTRACT

Local government is a basic mainstay for democratic Welfare States based on principle of maximum closeness to the citizen (subsidiarity). However, municipalities are faced with many problems to make this challenge, including precisely its limited financial capacity and infra municipalism. Therefore, they had to devise new ways to maximize administrative efficiency of tax administration and collection. Following the literature on models of local public service delivery, local tax management provision would be: direct supply by the local administration, delivery through special purpose bodies dependent on the local government, and outsourcing. These formulas, incorporating
} 
elements of both the New Public Management (NPM) and governance, have been reflected in various models ranging from inter administrative cooperation and supra local organizing skills. The aim of the study is to analyze these formulas for the sake of the principles of Solidarity, Equity and Redistribution. In this sense, municipalities have to maximize tax revenue for effective development of their social policies in the mode of Welfare States increasingly uncertain.

\section{KEYWORDS}

Local tax management, governance, solidarity, equity, financial sufficiency.

\section{CONTENTS}

1. Why we need to collect local taxes. 2. Management of local taxes. 3. Local Tax Administration framework. 4. Local Tax Administration in Spain. 5. Modernization of Local Tax Administration as support the Welfare State. 6. Conclusions. References.

\section{LA NECESIDAD DE RECAUDAR TRIBUTOS LOCALES}

La capacidad de recaudar tributos y responder de su gestión y utilización ante la ciudadanía, constituye uno de los principios democráticos esenciales, el de responsabilidad de los representantes ante el ciudadano contribuyente. Así se considera como la condición esencial para la existencia de una verdadera democracia local (INAP, 2002:138).

Además, la recaudación y gestión de los tributos locales resulta fundamental para el sostenimiento de las democracias locales. En primer lugar, porque estamos inmersos (y necesitamos) de un modelo expansivo de Estado del Bienestar, en el que el aumento del gasto público ha sido la tónica dominante en los últimos años $\mathrm{y}$, no parece que este pueda ser reducido tan fácilmente a pesar de la implantación de las políticas de austeridad, dada la profunda situación de crisis económica que está afectando a la situación social de tantos ciudadanos. Por otra parte habría que añadir, el reconocido, sostenido y creciente aumento de las expectativas de los ciudadanos con respecto a la calidad de la democracia local (Subirats, 1996:156), que se concretan en una demanda de más y mejores servicios y prestaciones.

Razonablemente, el Estado del Bienestar local solo se puede sostener con un equilibrado volumen de ingresos públicos (Garde, 1999:193) que se ajuste a los previsibles gastos sociales. La principal fuente de ingresos de los municipios son aquellos que provienen de los recursos que consiguen de sus tributos (Ministerio de Economía y Hacienda, 2011). Los ingresos tributarios de los Ayuntamientos son los que provienen de los cinco impuestos locales, el de bienes inmuebles (IBI), el de actividades económicas (IAE), el de vehículos de tracción mecánica (IVTM), el de incremento de valor de los terrenos de naturaleza urbana (IIVTNU, también conocido como plusvalía) y, el de construcciones y obras (ICIO), además de los que se derivan de las tasas y contribuciones especiales.

Lo que se pretende abordar en el presente trabajo es discernir cuál es la mejor vía para optimizar la recaudación y la gestión de los ingresos de derecho público tributario, sin trasladar ningún tipo de coste político, administrativo o de incremento impositivo a sus ciudadanos, y es más, que incluso contemple a los contribuyentes (ciudadanos o clientes) como eje vertebrador del servicio de gestión tributaria, que integra las facilidades para el efectivo cumplimiento de sus obligaciones tributarias. Consideramos por tanto que, la 
elección de la fórmula para llevar a cabo la gestión tributaria municipal es una de las decisiones estratégicas que deben adoptar los gobiernos locales para el desarrollo y mantenimiento de su Estado del Bienestar.

\section{LA GESTIÓN DE LOS TRIBUTOS MUNICIPALES}

La Constitución Española de 1978, rompiendo con el centralismo financiero y tributario de la etapa franquista (Carrillo, 1991), contempla expresamente los principios de autonomía y suficiencia financiera de los municipios (arts. 137 y 142), recogiendo que, las Haciendas locales "se nutrirán fundamentalmente de los tributos propios y de la participación en los del Estado y de las Comunidades Autónomas".

El principio de autonomía de las Entidades Locales, referido a los ingresos locales, entiende que éstas entidades tienen plena capacidad para decidir el volumen y la estructura de sus ingresos financieros, dado que el reconocimiento legislativo de potestades normativas locales sobre sus figuras impositivas, les permite -a los municipios y también a las provincias- el establecimiento y la aplicación de los tipos de gravamen en el territorio de la demarcación.

En cuanto al principio de suficiencia, se responsabiliza a las Corporaciones locales de la cifra de ingresos presupuestarios que obtendrán por medio de sus propios impuestos, de manera que la disposición de sus medios financieros para cubrir sus gastos no será solo responsabilidad del Estado y de las Comunidades Autónomas, sino que se comparte con todas las esferas de gobierno interesadas (Cayón, 2002:734).

Estos principios, evidentemente fueron desarrollados en las posteriores regulaciones que afectan a la financiación del régimen local como son la Ley 7/85, de 2 de abril, Ley Reguladora de las Bases del Régimen Local, la cual ha sido objeto de diversas modificaciones, entre ellas la operada por la Ley 57/2003, de 16 de diciembre, de medidas para la modernización del gobierno local -en estos momentos en proceso de revisión para adaptarla a el nuevo contexto político y administrativo- y, el R.D.L. 2/2004, de 5 de marzo, por el que se aprueba el Texto Refundido de la Ley Reguladora de las Haciendas locales que derogó la antigua Ley Reguladora de las Haciendas locales de 1989.

Sin embargo, la diversificación de las competencias de la gestión tributaria, a pesar de suponer un gran avance democrático para el ámbito local, plantea una serie de retos que superar para dar cumplimiento al art. 103.1 de nuestra Constitución, que ordena a las Administraciones Públicas cumplir con objetividad los intereses generales y actuar de acuerdo con los principios de eficacia, jerarquía, descentralización, desconcentración y coordinación (Lago, 1994:1568). Las principales dificultades con los que se encuentran los municipios para cumplir con efectividad y justicia sus competencias tributarias son: el endémico inframunicipalismo español, la infradotación de las administraciones locales, las resistencias en las relaciones interadministrativas y, la complejidad intrínseca del sistema tributario local.

En España, el régimen local se caracteriza principalmente por la diversidad de sus municipios, atendiendo a su tamaño, a su actividad económica o su localización geográfica. Según datos del Instituto Nacional de Estadística (INE) conforme a la información referida a las cifras de población al 1 de enero de 2012, existen en España 8.112 municipios, de los cuales 7.728 no alcanzan los 20.000 habitantes, lo que supone algo más del $95 \%$ de los municipios. Cabe decir que "un municipio por debajo de 20.000 
habitantes no es económicamente viable ni eficiente, y en España hay más de mil municipios con menos de 100 habitantes" (Tamames, 2012:191).

Tabla 1. Distribución de la población española en municipios

\begin{tabular}{|l|c|c|c|c|c|c|}
\hline \multicolumn{1}{|c|}{ ESPAÑA } & Municipios & $\%$ & \% acumulado & Población & $\%$ & $\%$ acumulado \\
\hline $\begin{array}{l}\text { Menos de 5.000 } \\
\text { habitantes }\end{array}$ & 6.796 & 83,77 & 83,77 & 5.988 .192 & 12,69 & 12,69 \\
\hline $\begin{array}{l}\text { Entre 5.000 y } \\
\text { 20.000 }\end{array}$ & 922 & 11,36 & 95,11 & 9.034 .186 & 19,14 & 31,83 \\
\hline $\begin{array}{l}\text { Entre 20.001 y } \\
\text { 50.000 }\end{array}$ & 185 & 2,28 & 97,39 & 7.499 .173 & 15,89 & 47,47 \\
\hline $\begin{array}{l}\text { Entre 50.001 y } \\
\text { 100.000 }\end{array}$ & 82 & 1,01 & 98,40 & 5.857 .700 & 12,41 & 60,13 \\
\hline $\begin{array}{l}\text { Entre 100.001 y } \\
\text { 500.000 }\end{array}$ & 57 & 0,7 & 99,03 & 11.186 .947 & 23,70 & 83,84 \\
\hline Más de 500.000 & 6 & 0,07 & 100 & 7.624 .295 & 16,16 & 100 \\
\hline TOTAL & \multicolumn{7}{|c|}{8.112} & & 47.190 .493 \\
\hline
\end{tabular}

Fuente: Elaboración propia a partir de datos del INE (Cifras del padrón a 1 de enero de 2010) en www.ine.es (Consulta: 08/09/2012)

En definitiva, este panorama de minifundismo local configurado por un número considerable de municipios con limitada dotación económica y capacidad de gestión y logística, que no les permite establecer "siquiera la turbadora sombra que toda administración que se precie debe estar en condiciones de proyectar" (Sosa Wagner, 2002:66), consideramos supone, el mayor obstáculo para la efectiva y eficaz gestión tributaria por los propios municipios.

El segundo problema a considerar, evidentemente relacionado con el anterior, sería la crónica infradotación de las Administraciones locales. Los Ayuntamientos iniciaron su camino democrático en un contexto de profunda crisis económica caracterizada por la stanflación (desempleo e inflación simultáneamente) en los que, a pesar de la falta de competencias directas en temas tales como políticas de empleo, de servicios sociales o de impulso económico, tuvieron que asumir todos ellos ante una ciudadanía que buscaba en el primer nivel administrativo, la respuesta a sus problemas (Subirats, 1996:159). Además, es más que constatable la tendencia que muestra que, la distribución de competencias en gestión tributaria a los municipios no se corresponde con una paralela asignación de medios personales y materiales. En lo que se refiere a las Corporaciones Locales, la insuficiencia de la dotación es una constante histórica (Cayón, 2006), y son conocidos como el "pariente pobre" de las nuevas instituciones democráticas (Olmeda, 2012) de tal forma que no se favorece, precisamente con la debida eficacia y eficiencia de los recursos, la asunción de las pesadas competencias gestoras en materia tributaria. En el mismo sentido, Parada (2007:34) incluso llega a considerar a los municipios "víctimas de la violencia de género institucional", dado el prolongado sufrimiento centralista al que han sido sometidos, primero por el Estado, y ahora por las Comunidades Autónomas.

El fraccionamiento y la estanqueidad de la información entre las administraciones suponen un gran problema para la correcta gestión administrativa de los organismos supeditados. En particular, la doctrina siempre ha denunciado reiteradamente la excesiva compartimentación y falta de comunicación entre los órganos tributarios (Fuentes 
Quintana, 1990). Es obvio que esta situación puede empeorar sensiblemente si los distintos órganos de gestión dependientes de distintas administraciones se convierten en departamentos estancos en los que no circula fluidamente la información que unos tienen y otros necesitan. Por ejemplo, si la relación entre el Ayuntamiento y la Dirección General del Catastro no es fluida y no está contrastada, dará lugar a importantes desajustes en la gestión y liquidación del IBI. Puede ocurrir lo mismo con la relación entre la Dirección General de Tráfico y el IVTM, y desde luego con la Agencia Estatal Tributaria en todo lo relacionado con los embargos. En cualquier caso, son vitales técnicamente e incluso institucionalmente accesibles, las herramientas que se consideran esenciales para luchar contra la indeseable incomunicación, como son las nuevas tecnologías y la promoción de una eficaz red de colaboración interadmistrativa.

Por último, la complejidad del sistema tributario local y, más concretamente su gestión, que requiere de una permanente actualización y adecuación de sus estructuras para ajustarse a las constantes modificaciones legales, así como a las distintas interpretaciones que los Tribunales puedan realizar, complica bastante, si no la hace materialmente imposible, la labor tributaria que tienen asignados los Ayuntamientos, en la que como se ha descrito anteriormente, en la mayoría de los casos, sus estructuras administrativas son pequeñas y poco diferenciadas, disponen de escasos recursos (económicos, humanos, tecnológicos...) y, no resultan demasiado ágiles pues son incapaces de aprovechar las oportunidades que les ofrecen las colaboraciones interadministrativas .

Estas complicaciones derivadas de la constitucionalmente reconocida competencia tributaria, tenían unas graves derivaciones sobre los contribuyentes de los municipios. Por un lado, porque la prestación de los servicios locales no satisfacían sus necesidades por la crónica falta de recursos. Y por otro, se añadía el tener que soportar una injusta distribución de la carga fiscal al encontrarse con una administración municipal incapaz de detectar el fraude tributario, con enormes dificultades para materializar los medios que hacen efectivo el pago de impuestos, carente de los recursos técnicos necesarios para transmitir al ciudadano la información para el cumplimiento de sus obligaciones y, dotada con personal poco profesionalizado y motivado para el desarrollo de esas funciones (Domínguez-Berrueta y López, 1994:1117) .

Pero, las indudables ventajas de la autonomía financiera para los entes locales, no puede quedar oscurecida por los inconvenientes que genera (Mira-Perceval, 2000:25). Máxime considerando que la aplicación del criterio de subsidiariedad o de mayor proximidad al ciudadano en el ámbito local permite mejorar las agregaciones de las preferencias ciudadanas (Canales y Pérez, 2002:25). "El principio de subsidiariedad establece que las tareas públicas han de realizarse al nivel más cercano posible al ciudadano y con el menor uso posible de poder público, en función del problema que requiere la actuación colectiva "(Villoría, 1996:212 citando a Hood y Shuppert, 1988:250). Por eso, previendo las complicaciones que la aplicación directa de las competencias tributarias podía acarrear a la mayoría de los municipios, el legislador contempló la posibilidad de delegación o encomienda de la gestión y recaudación de los tributos locales a otros entes particulares de ámbito superior.

En este sentido, el art. 106.3 de la LBRL prevé la delegación de las actividades de gestión, recaudación e inspección de los tributos locales y restantes ingresos de derecho público en otras administraciones locales de ámbito superior, articulado avalado también por la normativa recogida en el art. 7 del Texto refundido de la LHL. 
Recapitulando, a fin de salvar todas las barreras que, como consecuencia del inframunicipalismo, se derivan de la labor de gestión tributaria y recaudación por los propios municipios, se plantea legalmente la posibilidad de la delegación de las competencias en materia tributaria en una autoridad supramunicipal, y más concretamente, en la Diputación provincial o en la Comunidad Autónoma uniprovincial.

En cualquier caso, la LBRL (art. 85.2) señala que la competencia delegada al ente superior sólo puede ser desempeñada por gestión directa mediante una de las siguientes fórmulas:

a) Gestión por la propia entidad

b) Gestión por el Organismo Autónomo Local

c) Entidad Pública Empresarial Local

d) Sociedad Mercantil Local, cuyo capital sea de titularidad pública

Dado que el servicio público tributario local no tiene asociada contraprestación económica, quedaría pues descartada la posibilidad de Entidad Pública empresarial y la Sociedad Mercantil. Por lo tanto, solo restaría a la administración decidir cuál es la mejor forma de llevar a cabo esta gestión tributaria conforme a los principios de eficacia, eficiencia y economía.

Recapitulando con lo expuesto hasta el momento, las posibilidades que se abren en el modelo de delegación de las competencias tributarias a la administración supramunicipal serían: la gestión sin organismo especializado por la propia Administración que recibe la encomienda o delegación, el Organismo Autónomo especializado y, la modalidad de gestión indirecta que permita diluir las barreras impuestas por el art. 85.2 de la LBRL.

\section{EL PANORAMA DE LA GESTIÓN TRIBUTARIA LOCAL}

Tal y como se ha expuesto, en nuestro país, la gestión tributaria y recaudación de los impuestos locales, tasas, precios públicos y exacciones locales, puede realizarse por el

Tabla 2. Modalidades para la prestación del servicio de gestión tributaria local

\begin{tabular}{|c|c|c|c|}
\hline \multirow{6}{*}{$\begin{array}{l}\text { I: Las competencias tributarias las } \\
\text { presta el Ayuntamiento }\end{array}$} & \multicolumn{3}{|l|}{ I.1.Tradicional } \\
\hline & \multirow{2}{*}{$\begin{array}{l}\text { I.2.Empresa } \\
\text { colaboradora }\end{array}$} & \multicolumn{2}{|c|}{ I.2.1 Modelo total } \\
\hline & & \multicolumn{2}{|c|}{ I.2.2 M. parcial } \\
\hline & \multirow{3}{*}{$\begin{array}{l}\text { I.3.Organismo } \\
\text { Autónomo Local } \\
(\mathrm{OAL})\end{array}$} & \multicolumn{2}{|l|}{ I.3.1 OAL } \\
\hline & & \multirow{2}{*}{$\begin{array}{l}\text { I.3.2 Empresa } \\
\text { colaboradora }\end{array}$} & I.3.2.1 M. total \\
\hline & & & I.3.2.2 M. parcial \\
\hline \multirow{6}{*}{$\begin{array}{l}\text { II: Delegación de competencias de } \\
\text { Haciendas locales a la institución } \\
\text { provincial }\end{array}$} & \multicolumn{3}{|l|}{ II.1.Tradicional } \\
\hline & \multirow{2}{*}{$\begin{array}{l}\text { II.2.Empresa } \\
\text { colaboradora }\end{array}$} & \multicolumn{2}{|l|}{ II.2.1 M. total } \\
\hline & & \multicolumn{2}{|l|}{ III.2.2 M. parcial } \\
\hline & \multirow{3}{*}{$\begin{array}{l}\text { II.3.Organismo } \\
\text { Autónomo Provincial } \\
\text { (OAP ) }\end{array}$} & \multicolumn{2}{|c|}{$\begin{array}{l}\text { II.3.2 OAP ( Suma Gestión Tributaria } \\
\text { Diputación Provincial de Alicante ) }\end{array}$} \\
\hline & & \multirow{2}{*}{$\begin{array}{l}\text { II.3.2 Empresa } \\
\text { colaboradora }\end{array}$} & II.3.2.1 M. total \\
\hline & & & II.3.2.2 M. parcial \\
\hline $\begin{array}{l}\text { III: Delegación de competencias a la } \\
\text { Comunidad Autónoma (uniprovincial ) }\end{array}$ & \multicolumn{3}{|c|}{$\begin{array}{l}\text { Se reproducen las variantes de los casos anteriores añadiendo al } \\
\text { modelo los tributos autonómicos }\end{array}$} \\
\hline
\end{tabular}

Fuente: Elaboración propia a partir de LBRL y LHL 
propio Ayuntamiento, por delegación a la Diputación o, incluso se permite externalizar directamente el servicio, siendo muy frecuente la combinación de varias fórmulas, por delegación total o parcial $\mathrm{y}$, que contempla la creación de organismos autónomos de recaudación.

De esta forma el panorama de prestación del servicio de gestión y recaudación municipal quedaría plasmado en el cuadro anterior:

- El Servicio tradicional burocrático

La gestión tributaria y la función de recaudación la realiza el propio Ayuntamiento, la Diputación o Administración Autonómica con sus recursos y funcionarios. Si son las entidades supramunicipales, lo harán a través del convenio y las competencias delegadas por los primeros. La cesión que los Ayuntamientos realicen de sus competencias puede abarcar todos o algunos tributos locales $\mathrm{y}$, todos o algunos procedimientos de gestión y recaudación, en función del contenido del convenio suscrito. Como exponen Salvador y Ribó (2012), las administraciones que prefieren esta opción son aquellas que ya han introducidos reformas hacia la flexibilidad en sus estructuras de personal que los equipararía con el sector privado en lo que respecta a los recursos humanos. En cualquier caso, consideramos que esta afirmación debería ser matizada en el caso de que la gestión la realiza el propio Ayuntamiento, en el sentido que la decisión de cesión de competencias a la Diputación por los municipios es debida en gran medida a que disponen de menos recursos de personal.

- El Servicio a través de empresa colaboradora (externalizado o outsourcing ).

Aunque existen argumentos críticos a la actuación de "empresas asesoras externas privadas colaboradoras en el procedimiento tributario y recaudatorio municipal", especialmente a lo que se refiere a su ámbito legal, se ha recogido esta fórmula en el esquema propuesto pues es una manifestación constatable de la realidad a la que se acogen los municipios. El razonamiento en su contra se basa en las siguientes premisas: "esas empresas no solo llevan a cabo materialmente tareas reservadas por ley a funcionarios públicos (aunque formalmente el recaudador sea el funcionario municipal ), lo que va en contra de la LBRL, sino que con escaso o nulo control municipal disponen y manejan unas bases de datos de ciudadanos que requerirá una pronta intervención de la gerencia de la Agencia de Protección de Datos, al incluirse en ellas la relación de todos los propietarios de inmuebles y vehículos (a través del IBI, de las licencias de apertura, de la basura industrial o del ICIO ), de determinados negocios jurídicos (transmisiones de inmuebles a través del IIVT o del IBI ), de las cuentas corrientes ( a través de las domiciliaciones o de los embargos de cuentas) etc. ... El hecho aún más grave cuando detrás de estas empresas hay entidades financieras, algunas de las cuales ofrecen directa o indirectamente "servicios recaudatorios" a los Ayuntamientos: ¿Quién garantiza la confidencialidad de los datos?, ¿Es normal que tengan acceso total al registro de propiedades inmuebles, de vehículos, de actividades económicas, etc.? ¿No utilizarán la información de morosidad con los Ayuntamientos como referencia para sus relaciones con sus clientes?, ¿Embargarán, vía funcionario municipal, por supuesto, las cuentas de sus propios clientes? Son preguntas que plantean importantes dudas "(Rueda, 2001:18).

En cualquier caso, la externalización de servicios públicos en una constante en los modelos de modernización administrativa (Goldsmith y Edgers, 2004), (Wollman, 2003) y (Kelly, 1998), y de hecho están muy extendidos. Si bien esta circunstancia no debilita el argumento que "la externalización no deroga la responsabilidad de la Administración por el servicio prestado" y que "son insustituibles las tareas de inspección y vigilancia", 
cuando se pretende que la externalización no se convierta en un mero traspaso de funciones a terceros ni una alternativa al desentendimiento, situación muy tentadora, a los extensos problemas de lo público (Olías de Lima, 2011:2).

Por ello, en el caso de la posible externalización de los servicios tributarios locales, conforme al art. 85.3 de la LBRL, la gestión ha de estar controlada en última instancia por la Administración, quedando pues las actuaciones de las empresas colaboradoras limitadas a aquellas que no impliquen ejercicio de autoridad.

Así, aparecen dos posibilidades:

- Colaboración total: la empresa colaboradora aporta el Know How, personal, recursos informáticos e incluso instalaciones.

- Colaboración parcial: la empresa colaboradora solo proporciona parte del servicio a la administración conforme a lo designado en el contrato de colaboración (en competencias y tributos) quedando el resto en manos de ésta.

Según Salvador y Ribó (2012), no queda claro que la externalización suponga un verdadero cambio en el modelo de gestión tributaria, a pesar de las reconocidas presiones externas a la búsqueda de alternativas a la prestación directa. Las administraciones que suelen optar por esta fórmula son las que se caracterizan por el stress fiscal y su cercanía a las áreas metropolitanas, pues es dónde existe mayor densidad de empresas que ofrecen estos servicios.

- El Organismo Autónomo.

Las razones para la adopción de esta fórmula puede esquematizarse en dos: las de carácter administrativo-técnico que se deducen de la aplicación de una fórmula de descentralización funcional, y las de carácter eminentemente financiero para proveerse de mayor autonomía presupuestaria y mejora de los criterios de eficiencia, aunque ésta ha de demostrarse, pudiendo ser probada mediante su capacidad para generar ingresos propios para su sostenimiento (Martínez-Alonso, 2007:135). La creación de un Organismo Autónomo ad hoc específico para la gestión tributaria y recaudación de los ingresos municipales se guía por la búsqueda de una mayor flexibilidad en la gestión y organización de las estructuras pero manteniendo el control y la titularidad pública. Estos organismos están regulados por la LBRL y por el RDL 781/1986, aunque norma fundamental serán sus Estatutos. Se permite, a su vez, las siguientes fórmulas:

- Servicio desarrollado completamente por el organismo autónomo.

- Servicio prestado conjuntamente junto con empresa colaboradora (cesión total o parcial del servicio). Ejemplo: La administración pone los recursos humanos (funcionarios) pero los recursos técnicos son comprados o desarrollados por empresas privadas.

En general, los organismos autónomos pretenden representar lo mejor y evitar lo peor, tanto del ámbito público como del privado. Así, mantienen la financiación pública con sus correspondientes controles $\mathrm{y}$, en ocasiones, mantienen ciertos privilegios públicos; mientras que al tratar de evitar los controles burocráticos, permiten el desarrollo de fórmulas de gestión privada, organizativamente más flexibles. El anhelo de los organismos autónomos es la búsqueda de organizaciones independientes y no controlada por el gobierno para gestionar servicios públicos de neutralidad partidista (Villoria, 1996:209). Además, en nuestro caso, la gestión y recaudación de los tributos locales, puede resultar muy interesante para los gobernantes y responsables locales la presencia de un organismo neutral que se encargue de esta tarea y los libere de las presiones directas de sus ciudadanos. 
Tabla 3. Panorama provincial de la gestión tributaria local en España

\begin{tabular}{|c|c|c|c|c|c|}
\hline Provincia & Modalidad de delegación & $\mathrm{N}^{\circ}$ Aytos. & \begin{tabular}{|l|}
$\mathrm{N}^{\circ}$ Aytos. que \\
delegan
\end{tabular} & $\begin{array}{l}\text { Delegación de la capital } \\
\text { provincia }\end{array}$ & $\begin{array}{l}\text { Empresa } \\
\text { colaboradora }\end{array}$ \\
\hline Álava & Diputación Foral & 51 & - & No & \\
\hline Albacete & OAP & 87 & 87 & $\mathrm{Si}$ & $\overline{\mathrm{Si}}$ \\
\hline Alicante & OAP & 141 & 141 & Parcial. Solo ejecutiva & No \\
\hline Almería & Diputación & 102 & - & $\mathrm{No}$ & $\mathrm{Si}$ \\
\hline Asturias & Ente Público & 78 & 76 & No & $\mathrm{Si}$ \\
\hline Ávila & OAP & 248 & - & No & $\mathrm{Si}$ \\
\hline Badajoz & OAP & 164 & 164 & $\mathrm{Si}$ & No \\
\hline Baleares, Islas & Comunidad Autónoma & 67 & & & \\
\hline Barcelona & OAP & 311 & 308 & No & No \\
\hline Bizkaia & Diputación Foral & 112 & 103 & No & No \\
\hline Burgos & Diputación & 371 & 366 & No & - \\
\hline Cáceres & OAP & 221 & 220 & $\mathrm{Si}$ & No \\
\hline Cádiz & Diputación & 44 & - & - & No \\
\hline Cantabria & Comunidad Autónoma & 102 & - & - & - \\
\hline Castellón & OAP & 135 & 135 & $\mathrm{Si}$ & $\mathrm{Si}$ \\
\hline Ciudad Real & OAP & 102 & - & - & - \\
\hline Córdoba & OAP & 75 & 72 & No & - \\
\hline Coruña, $\mathrm{A}$ & Diputación & 94 & 49 & No & $\mathrm{Si}$ \\
\hline Cuenca & OAP & 238 & 202 & No & - \\
\hline Gipuzkoa & Diputación Foral & 88 & 86 & No & $\mathrm{Si}$ \\
\hline Girona & OAP & 221 & 209 & No & No \\
\hline Granada & OAP & 168 & 144 & No & $\mathrm{Si}$ \\
\hline Guadalajara & Diputación & 288 & 270 & No & $\mathrm{Si}$ \\
\hline Huelva & OAP & 79 & 79 & $\mathrm{Si}$ & - \\
\hline Huesca & OAP & 202 & 194 & No & - \\
\hline Jaén & Diputación & 97 & 62 & No & - \\
\hline León & Diputación & 211 & 140 & No & $\mathrm{Si}$ \\
\hline Lleida & OAP & 231 & 226 & No & No \\
\hline Lugo & Diputación & 67 & 63 & No & - \\
\hline Madrid & Local & 179 & - & - & $\mathrm{Si}$ \\
\hline Málaga & OAP & 101 & 95 & No & - \\
\hline Murcia & OAP & 45 & 32 & No & - \\
\hline Navarra & Local & 272 & - & - & - \\
\hline Ourense & Diputación & 92 & 81 & No & - \\
\hline Palencia & OAP & 191 & 185 & No & $\mathrm{Si}$ \\
\hline Palmas, Las & OAP & 34 & 19 & No & $\mathrm{Si}$ \\
\hline Pontevedra & OAP & 62 & 62 & $\mathrm{Si}$ & $\mathrm{Si}$ \\
\hline Rioja, La & Comunidad Autónoma & 174 & 168 & No & $\mathrm{Si}$ \\
\hline Salamanca & OAP & 362 & 362 & $\mathrm{Si}$ & No \\
\hline Sta.Cruz Tenerife & OAP & 54 & 26 & No & $\mathrm{Si}$ \\
\hline Segovia & Diputación & 209 & 139 & No & - \\
\hline Sevilla & OAP & 105 & 102 & No & $\mathrm{Si}$ \\
\hline \begin{tabular}{|l|} 
Soria \\
\end{tabular} & OAP & 183 & 180 & No & - \\
\hline Tarragona & OAP & 184 & 184 & No & No \\
\hline Teruel & Diputación & 236 & - & No & - \\
\hline Toledo & OAP & 204 & 199 & No & $\mathrm{Si}$ \\
\hline Valencia & Diputación & 266 & 231 & No & $\mathrm{Si}$ \\
\hline Valladolid & OAP & 225 & 221 & No & $\mathrm{Si}$ \\
\hline Zamora & Diputación & 248 & 180 & No & $\mathrm{Si}$ \\
\hline Zaragoza & Diputación & 293 & 282 & No & $\mathrm{Si}$ \\
\hline
\end{tabular}

Fuente: Elaboración propia a partir de datos del INE, 2010 y de la páginas Webs de las Diputaciones y organismos provinciales de gestión tributaria y recaudación. Actualización: 18/09/2012 


\section{EL HORIZONTE DE LA GESTIÓN TRIBUTARIA LOCAL EN ESPAÑA}

El panorama de la gestión tributaria nos ofrece una compleja y heterogénea radiografía a lo largo de todo el territorio español.

No existe un único modelo válido para todas las provincias $\mathrm{y}$, mucho menos para todos los municipios, aunque sí podría generalizar que los municipios españoles tienden mayoritariamente a delegar sus tributos en el organismo provincial y, éste a su vez, suele preferir la fórmula del organismo autónomo (OAP). Como se ha comentado, resultan irreconciliables las razones que dificultan la gestión tributaria por el propio municipio, a lo que habría que añadir las economías a escala crecientes que produce la delegación en la organización provincial que gestiona las competencias de todos o gran parte del territorio. Las economías a escala resultan evidentes en aspectos como personal, aplicaciones tecnológicas, relaciones con otras administraciones y colegios profesionales, información y publicidad, etc.

Aún así, la diversidad sigue siendo muy extensa, no solo marcada por la amplitud de opciones que se abre a la gestión en el organismo autónomo sino también porque mayoritariamente, las capitales de provincia no suelen delegar en la institución provincial. Considero que esta misma heterogeneidad es la que determina que el escenario de colaboración sea idóneo para compartir experiencias entre las distintas agencias $u$ organismos especializados en la gestión tributaria local.

De este variado panorama que ofrece la gestión tributaria local, nos permitimos en cualquier caso avanzar las siguientes tendencias de actuación:

1) Aproximadamente el $90 \%$ de los municipios españoles tienden a delegar algún aspecto relacionado con la gestión de sus tributos en su organismo provincial. Resulta particularmente destacable que son los municipios que constituyen las capitales de provincia, los más reacios a la cesión de sus competencias tributarias, apenas son 7 los que han delegado la gestión de sus tributos en otra institución (Albacete, Alicante, Badajoz, Cáceres, Pontevedra, Huelva y Salamanca). Una posible explicación interna radica en la resistencia que ejercen los técnicos de recaudación de las instituciones locales mayores a la delegación supramunicipal. También mientras que la administración local más pequeña puede estar interesada en trasladar la imagen a sus ciudadanos que la recaudación de impuestos se hace desde otro organismo, mientras que ellos son los proveedores de los servicios, las administraciones de mayor tamaño también les interesa ser visualizadas políticamente como responsables de sus ingresos públicos.

2) El organismo provincial suele preferir que la gestión tributaria delegada se realice a través de un organismo autónomo provincial (existen 27 organismos autónomos provinciales creados ad hoc para la gestión tributaria).

3) Las empresas colaboradoras aparecen prestando asistencia tanto a los servicios dependientes de las Diputaciones como en los organismos autónomos provinciales. Los tipos de servicio que pueden prestar la empresa colaboradora van desde el desarrollo y mantenimiento de una herramienta informática especializada hasta la aportación de personal especializado que realice la gestiones de cobro y después trasfiera el dinero. Se deduce que en estos casos el modelo desarrollado es el subsidiario frente al modelo integral de competencias en el que no se externaliza el servicio.

4) Las comunidades autónomas uniprovinciales han optado tanto por la concentración de la gestión tributaria autonómica y local, como por dejar a los municipios que se 
organicen sus propias competencias sin constituir ningún servicio que lo gestione (en Madrid y Navarra).

5) Las evidencias parecen indicar que la utilización de uno u otro modelo no depende tanto de la ideología o color político del gobierno sino de su efectividad. En cualquier caso, en la toma de la decisión, los responsables políticos valoran, además de los criterios para la toma de decisión de la delegación, las repercusiones derivadas de la gestión del cambio y los factores de oposición a éste que pueden reflejarse en las posibles actuaciones de los sindicatos o la competencia.

\section{LA MODERNIZACIÓN DE LA GESTIÓN TRIBUTARIA LOCAL COMO SUSTENTO DEL ESTADO DEL BIENESTAR}

Los municipios juegan un papel fundamental en el Estado del Bienestar por la aplicación del principio de proximidad y cercanía al ciudadano. Además soportan la sobrecarga de competencias (especialmente las denominadas impropias) transferidas al ámbito local desde los niveles de gobierno superiores y/o exigidas por sus propios ciudadanos además de las crecientes demandas ciudadanas que, tienen en el nivel local la primera referencia a sus expectativas (Canales, 2010). Es por ello que resulta de importancia maximizar la eficacia de los ingresos públicos locales ante un panorama en el que las necesidades de gasto público crecen incesantemente (Suárez, 2008).

Los ingresos públicos que reciben los gobiernos locales provienen fundamentalmente de la participación de los ingresos del Estado y de las subvenciones de las Comunidades autónomas y, más especialmente de sus recursos tributarios. Así, interesa que estos recursos tributarios se canalicen de la forma más eficientemente posible tanto desde un punto de vista de equilibrio económico como político. El coste de una gestión tributaria ineficiente y de la percepción generalizada de inequidad tributaria resulta altísimo para cualquier democracia que se precie. Así, los municipios deben encontrar fórmulas que al mismo tiempo que evitan cualquier tipo de evasión o fraude fiscal, facilitan el cumplimiento de las obligaciones tributarias de los ciudadanos. Interesa por tanto encontrar organismos especializados en gestión tributaria local que realice estas gestiones. De las distintas posibilidades que se han propuesto a los largo de este trabajo, que se lleve a cabo por la propia entidad, que se delegue a la Diputación o que se externalice el servicio en una empresa colaboradora privada, interesará aquel que mejor pueda llevar a cabo las siguientes recomendaciones (OCDE, 2009).

a) Dar facilidades a los ciudadanos en el pago de sus tributos locales mediante la concesión de aplazamientos y fraccionamientos de sus obligaciones tributarias. La administración tradicional de tipo burocrático suele tener problemas para realizar esta recomendación, pues las rigideces y los protocolos de actuación cuanto menos, complican el procedimiento que, a todas luces debería ser muy simple y accesible. Por ese motivo y atendiendo a esta importante solicitud, unida a una mayor demanda de los contribuyentes a estas facilidades relacionadas con el pago en periodo voluntario, consideramos como más adecuadas las opciones que flexibilizan los procedimientos. Así la descentralización funcional, a través de un organismo autónomo es una opción muy interesante. Podría ser discutible si la recaudación realmente aumenta o disminuye con este tipo de medidas, pero lo que sí que queda claro es que a los ciudadanos se le facilita el cumplimento de sus obligaciones, percibiendo así una mayor justicia y solidaridad tributaria. 
b) Aplicar las nuevas tecnologías tanto para mejorar la propia gestión interna y el tratamiento de los datos que se manejan sobre el contribuyente sino también para ampliar los canales de relación con los ciudadanos (pago vía telemática, información sobre trámites...). La Nueva Gestión Pública (NGP) como medida modernizadora revaloriza el papel del ciudadano-cliente situándolo como protagonista indiscutible de lo público. Aunque si bien en estos momentos las implicaciones de conceptualizar como cliente a los ciudadanos contribuyentes puede resultar discutible (Román, 2012), la realidad es que el uso de las Nuevas Tecnologías de la Información y Comunicación (NTCIs) están totalmente presente en cualquier ámbito de la sociedad, por lo que parece completamente lógico que se traslade al ámbito de la Administración Pública, en consonancia con la NGP y el modelo de Gobernanza. De nuevo, resultan más operativas las fórmulas organizativas que flexibilizan y mantienen la actualización en el uso de las NTCIs.

c) A los gobiernos locales les interesa gestionar sus impuestos mediante unas fórmulas flexibles que les permitan obtener recursos financieros mensualmente, en definitiva, los anticipos a su recaudación. La recaudación de los impuestos y las tasas municipales se realiza en unos periodos de tiempo muy concretos, mientras que los municipios necesitan disponer de fondos durante todo el ejercicio. Es por ello que resulta interesante que los organismos de gestión tributaria puedan actuar como banca local facilitando crédito sobre los ingresos previstos a partir de los denominados "anticipos a la recaudación". Esos anticipos tienen como finalidad ayudar a la planificación económica de los municipios durante todo el ejercicio.

d) Mejorar los niveles de recaudación sin dejar de lado que, los ciudadanos precisan de una atención de más calidad basada en la excelencia. El Modelo de calidad total para la gestión tributaria considera las siguientes actuaciones: la excelencia en la atención a los ciudadanos (la reducción de los tiempos de espera y el feedback en las quejas y sugerencias, son elementos centrales para su consecución), minimizar los desplazamientos al ciudadano contribuyente (no solo a través de las oportunidades que ofrecen las TIC's sino también mediante la racionalización y simplificación de trámites ), la reducción de los plazos de resolución de los plazos administrativos, las ya comentadas facilidades para el pago de tributos mediante aplazamientos y fraccionamientos de la deuda, y la autoevaluación de la organización como instrumentos de la mejora continua.

e) Para finalizar, consideramos que el aprovechamiento de las economías a escala que llegan a producir las fórmulas de gestión de los tributos desde el ámbito provincial son fundamentales, no solo para el sostenimiento de un Estado del Bienestar que emplea menos recursos (y por consiguiente está reduciendo el gasto público sin ningún coste social) sino también, porque se optimizan las prestaciones en base a los principios de equidad y justicia tributaria. También creemos que constituye un sencillo mecanismo para abordar las profundas desigualdades que entre los municipios existe en España, siendo estas más frecuentes y profundas que entre las regiones (Alarcón y Peñarubia, 2012:25). De esta forma, consideramos que los organismos con capacidad para aplicar todas las recomendaciones expuestas y evitar los problemas señalados, estarán maximizando la ecuación biunívoca entre Democracia y Estado del Bienestar. 


\section{CONCLUSIONES}

-La gestión tributaria local es una actividad altamente compleja dado el cambiante y variado marco legal que lo sustenta, las garantías que se asocian a la delicada información personal que se manejan sobre los contribuyentes, la dificultad para encontrar y mantener un personal cualificado y actualizado en la materia, el sofisticado entramado tecnológico para optimizar el tratamiento de los datos, las necesidades económicas y financieras de los municipios... etc.

-Además, la gestión tributaria y recaudación se realiza en un entorno caracterizado por el infra municipalismo, la fragmentación y la heterogeneidad, lo que incrementa las dificultades propias de su gestión.

-Es por ello, que parece conveniente, y así lo contempla la legislación local, establecer unos mecanismos de coordinación intermunicipal para el desarrollo de las sinergias tributarias. Estos mecanismos se concretan en la delegación de sus competencias tributarias en organizaciones supramunicipales: en la propia Diputación (o Comunidad Autónoma uniprovincial), en Organismo autónomo especializado o en empresa externa colaboradora.

-En cualquier caso, la delegación de las competencias locales tributarias no obedece tanto a una intención de modernización sino más bien a una necesidad funcional y operativa.

-A su vez, no existe un único modelo consensuado y generalizado por la totalidad de municipios españoles, lo que ha provocado una importante disparidad de fórmulas de gestión y recaudación con resultados diversos.

-Este entorno tan diversificado ha promovido experiencias colaborativas y de modernización administrativa en la búsqueda de organizaciones más flexibles y, que guiadas por los principios de eficacia, eficiencia y economía de sus resultados, no se alejen del eje que una mejor administración, y en concreto que, una mejor administración tributaria local conduce a unos ciudadanos más satisfechos con la ecuación sobre lo que pagan y lo que reciben, y en definitiva, se estará profundizando en la calidad de nuestra democracia.

-Por otra parte, la idiosincrasia de la "nueva forma de gobernar" que propone el modelo de "gobernanza", extiende los parámetros reformistas de la Nueva Gestión Pública (NGP) a la participación de los actores implicados y la gestión de redes. En este sentido, la gestión tributaria local ha de buscar el logro de los principios de equidad y equilibrio tributario combinados con la ética democrática.

-Para finalizar, el reto de la gestión tributaria local en los Estados de Bienestar en tiempos de crisis consistirá en compatibilizar las clásicas E's relacionadas con la Economía, Eficiencia y Eficacia con otras, como son la Ética, el Equilibrio y la Equidad. La fórmula exitosa será aquella que permita maximizar la ecuación con estas variables.

\section{BIBLIOGRAFÍA}

ALARCÓN, G. y PEÑARRUBIA, D. (2012), "El nuevo IBI en España y la progresividad, más cerca" en Más Poder Local, no 13, pp.24-25.

CANALES, J.M y PÉREZ, P.L. (2002), Introducción al Gobierno y a la Gestión Local, Alicante, Editorial club Universitario (ECU). 
CANALES, J.M. (2010), "El nuevo papel del territorio y su incidencia en los gobiernos locales: algunos apuntes sobre el caso español" en Politeia, Revista del Pensamiento Político, $\mathrm{n}^{\circ}$ 48, pp. 1521.

CARRILLO, E. (1991), Gestión de recursos humanos, presupuestación y hacienda local en España, Madrid, Instituto de Estudios Fiscales.

CAYÓN, A. (dir.) (2002), Los Impuestos en España, Navarra, Aranzadi.

DOMÍNGUEZ-BERRUETA, M. y PÉREZ, V. (1994), "La gestión de los servicios económicos municipales. Un nuevo proyecto. El organismo autónomo de gestión económica y recaudación del Ayuntamiento de Salamanca" en A. Pérez Moreno (coord.) Administración Instrumental. Libro homenaje a Manuel Francisco Clavero Arévalo, Madrid, Civitas, pp. 1117-1133.

FEMP (2006), La situación de los Ayuntamientos en España. Sus carencias económicas y sus problemas de gestión: diagnóstico y propuestas en una perspectiva Europea, Madrid, FEMP.

FUENTES QUINTANA, E. (1990), La reforma fiscal y los problemas de la Hacienda Pública Española, Madrid, Civitas.

GARDE ROCA, J.A. (1999), "Sistema fiscal y modelo social: el alcance de las últimas reformas" en J.A. Garde Roca (ed.), Políticas sociales y Estado de Bienestar en España, Madrid, Editorial Trotta, pp.191-221.

GOLDSMITH, S. y EGGERS, W.D (2004), Governing by Network, Washington DC, The Brookings institution.

INAP (ed.) (2001), Reorganizar la acción pública local. Informe Mauroy. Comisión para el futuro de la descentralización, Documentos INAP, $\mathrm{n}^{\circ}$ 26, Madrid, INAP.

KELLY, K. (1998), New Rules for the New Economy: 10 Radical Strategies for a Connected World, New York, Viking Penguin.

LAGO, J.M. (1994), "Algunas consideraciones sobre la distribución de competencias de gestión tributaria en España" en El sistema económico de la Constitución española, Madrid, Dirección General del Servicio Jurídico del Estado, Ministerio de Justicia. Secretaria General Técnica, pp. 1562-1583.

MARTÍNEZ-ALONSO, J.L. (2007), "Medidas de mejora en el funcionamiento de las personificaciones instrumentales para la gestión de los Servicios Públicos locales" en Revista de Estudios Locales (Cunal), $\mathrm{n}^{\mathrm{o}}$ 1, pp. 133-156.

MINISTERIO DE ECONOMÍA Y HACIENDA (2011), Las haciendas locales en cifras. Año 2009, Madrid, MEH.

MIRA-PERCEVAL, A. (2000), "La cooperación insular y provincial en materia de gestión tributaria" en Análisis Local, no 30, pp. 23-28.

OCDE (2009), Foro sobre la Administración Tributaria. Subgrupo formado para la información a los contribuyentes, Paris, OECD.

OLMEDA, J. A. (2012), "Los gobiernos locales" en J.A. Olmeda, (et al.) (dir.), Las Administraciones Públicas en España, Valencia, Tirant lo Blanch, pp.173-215.

PARADA, J.R. (2007), La segunda descentralización: del estado autonómico al municipal, Navarra, Aranzadi.

RUEDA, S. (2001), "Las Diputaciones y la gestión tributaria y recaudatoria local (I) y (II)" en Cuadernos de Gestión Pública Local. Instituto Superior de Economía Local (ISEL), $2^{\circ}$ semestre 2000 y $2^{\circ}$ semestre 2001 , pp. 28-42.

RUIZ-HUERTA, J. y VILLORIA, M. (dirs.) (2010), Gobernanza democrática y fiscalidad, Madrid, Tecnos.

ROMÁN, L. (2012), "Consecuencias políticas de la acción colectiva: el paradigma del ciudadano cliente" en Revista de Investigaciones Políticas y Sociológicas RIPS, vol.11, $\mathrm{n}^{\circ}$ 1, pp.81-104.

SALVADOR, M. y RIBÓ, C. (2012), "Circumventing bureaucracy: service delivery choices in Spanish municipal administrations", paper del XXII Congreso Mundial de Ciencia Política, Madrid.

SOSA WAGNER, F. (2002), "La administración como ingrediente del Estado", en Papeles de Economía Española, n ${ }^{\circ}$ 92, pp.58-67.

SOSA WAGNER, F. (2004), La gestión de los servicios públicos locales, Madrid, Civitas. 
SOSA WAGNER, F. y SOSA MAYOR, I. (2007), El Estado fragmentado. El modelo austrohúngaro y brote de naciones en España, Madrid, Trotta.

SUAREZ, J. (coord.) (2008), La financiación local en España: radiografía del presente y propuesta de futuro, Salamanca, FEMP.

SUBIRATS, J. (1996), "Análisis de políticas públicas y gestión pública: promesas y riesgos de una vida en común", en J. Subirats y J. Brugué, Lecturas de gestión pública, Madrid, INAP, BOE, pp. 151-162.

TAMAMES, R. (2012), La crisis de la deuda soberana en la Eurozona: España, un proyecto de país, Madrid, Turpial.

VILLORIA, M. (1997), La modernización de la administración como instrumento al servicio de la democracia, Madrid, BOE.

WOLLMANN, H. (2003). "Coordination in the Intergovernmental Setting "en G. Peters y J. Pierre, Handbook of Public Administration, London, SAGE, pp. 594-606.

\section{Breve currículo}

\section{Irene Belmonte Martín}

Profesora Colaboradora del área de Ciencia Política y de la Administración de la Universidad Miguel Hernández. Su línea de investigación principal se desarrolla sobre la base de la modernización de la administración pública, especialmente en lo que se refiere a la adaptación de la gestión pública local a la nueva gobernanza. Otras líneas de su trabajo se encuadran en el análisis de las posibilidades de las NTICS aplicadas al gobierno, el estudio de los sistemas electorales y sus consecuencias sobre el sistema político y, la evaluación de las políticas públicas de igualdad. 